ML204
inhibits
intracellular
rises in $\mathrm{Ca}^{2+}$
in response
to LPS or PS,
and protects
podocytes
from
cytoskeletal
changes.

\section{Targeting a faulty filter}

Albuminuria, the spillage of plasma albumin into the urine, is caused by defective blood filtration in the kidney. It is often a consequence of cardiovascular or metabolic disease, and there are no specific therapies for this condition. Now, writing in the Journal of Clinical Investigation, Anna Greka and colleagues demonstrate that the transient receptor potential channel 5 (TRPC5) is involved in the pathogenic remodelling of the kidney filter, and show that a small-molecule inhibitor of TRPC5 can protect mice from pharmacologically induced kidney injury. cells called podocytes extend

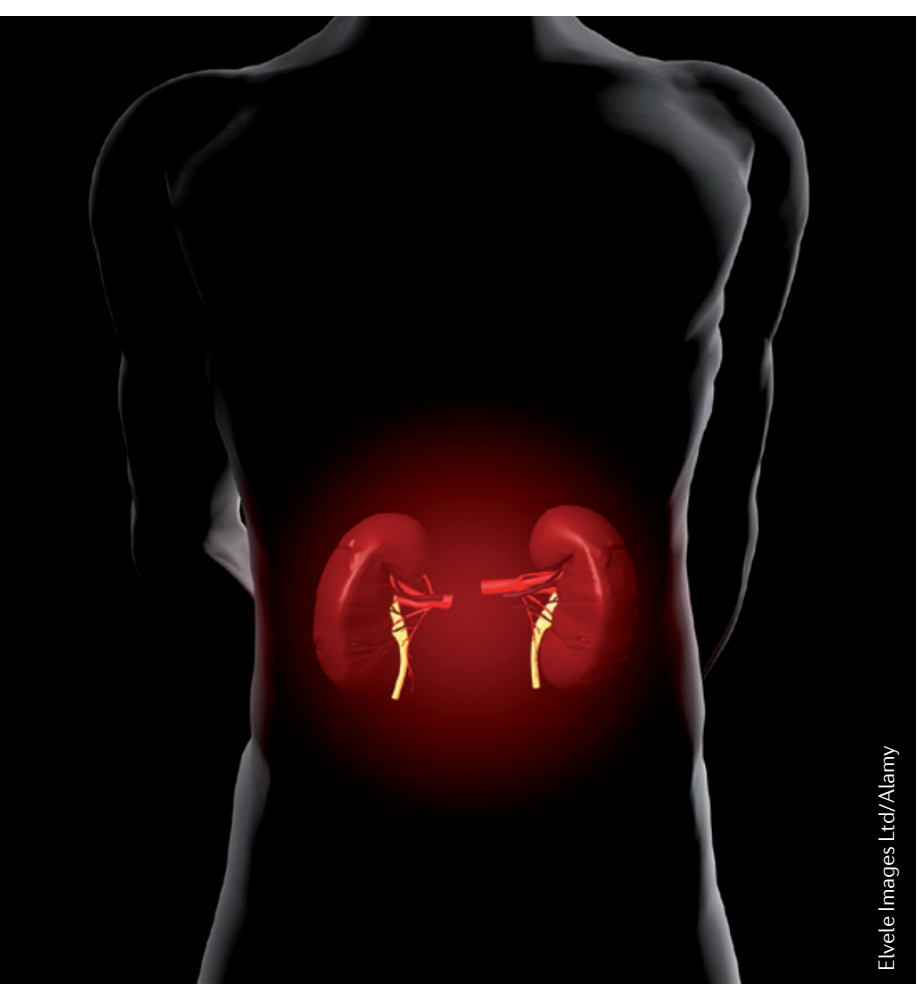

The human kidney filter, located in the glomerular and peritubular capillaries in the kidney, is essential for sorting molecules for passage into the urine or retention in the bloodstream. Specialized kidney microtubule-based major processes and actin-based foot processes (FPs) around the capillaries, and protect glomerular filter function. Podocyte injury can lead to albuminuria, and an early (and reversible) step in podocyte injury is the simplification and retraction of their FPs, a process called FP effacement (FPE). This is thought to occur in response to disrupted $\mathrm{Ca}^{2+}$ signalling.

To develop potential therapeutic strategies for treating albuminuria, the authors sought to identify the $\mathrm{Ca}^{2+}$ channel responsible for podocyte remodelling. They focused on TRPC5 as this ion channel is highly expressed in the kidney. Furthermore, $\mathrm{Ca}^{2+}$ influx through TRPC5 is known to modulate the GTPase RAC1 and lead to the disruption of the actin cytoskeleton, and constitutively active RAC1 has been shown to cause albuminuria in mice.

Immunolocalization studies confirmed the presence of TRPC5 in podocytes. Interestingly, TRPC5-knockout mice were shown to be resistant to lipopolysaccharide (LPS)-induced albuminuria - a model that reflects the early steps in filtration barrier damage. As opposed to wild-type mice, which have a severely damaged glomerular architecture and FPE in response to LPS injection, TRPC5-knockout mice did not show such structural changes. This result was confirmed in a second model of albuminuria, induced by the injection of protamine sulphate (PS). In ex vivo experiments using isolated glomeruli, the authors showed that both LPS and PS evoke a rise in $\mathrm{Ca}^{2+}$ in wild-type podocytes, which is markedly diminished in TRPC5-knockout podocytes.

Next, the authors tested the recently identified small-molecule inhibitor ML204, a reversible pore blocker of TRPC5, in cultured podocytes and in isolated mouse glomeruli. Biochemical analysis as well as real-time live confocal imaging demonstrated that ML204 inhibits intracellular rises in $\mathrm{Ca}^{2+}$ in response to LPS or PS, and protects podocytes from cytoskeletal changes by preventing the TRPC5dependent activation of RAC1. In vivo, continuous perfusion of mice with ML204 conferred significant protection from PS-induced FPE and intraperitoneally injected ML204 protected mice from LPS-induced albuminuria.

These results reveal TRPC5 inhibition as a specific strategy to protect the glomerular filter barrier in the early stages of barrier damage - which, as the authors point out, is particularly important given the rapidly growing epidemic of metabolic disease.

Alexandra Flemming

ORIGINAL RESEARCH PAPER Schaldecker, T. et al. Inhibition of the TRPC 5 ion channel protects the kidney filter. J. Clin. Invest. 123, 5298-5309 (2013) 